\title{
ENSAIO DE MOLHABILIDADE COMO INDICATIVO DA POTENCIALIDADE DE UTILIZAÇÃO DE RESÍDUO DE ROCHAS ORNAMENTAIS EM CERÂMICA DE REVESTIMENTO
}

\author{
Tiago Marvila Gomes ' \\ Simone Pereira Taguchi Borges ${ }^{2}$ \\ Mirna Aparecida Neves \\ Luiz Antônio Borges Junior ${ }^{2}$
}

\section{Resumo}

A produção de placas de rochas ornamentais gera uma significativa quantidade de resíduos. A composição destes resíduos indica a possibilidade de seu aproveitamento em vários produtos, como, por exemplo, na fabricação de cerâmica de revestimento. $\mathrm{O}$ objetivo deste trabalho foi estudar a molhabilidade de material vidrado sobre cerâmica vermelha, ambos compostos com resíduos de rochas ornamentais. Foram realizados ensaios de molhabilidade pelo método da Gota Séssil, que envolve a fusão de corpos de prova de vidrado sobre placas de cerâmica vermelha sinterizadas. $O$ ângulo de contato foi medido durante a fusão do vidrado e os resultados foram analisados utilizando análise fatorial $2^{2}$. Os resultados mostraram que os resíduos podem ser usados na fabricação de cerâmica de revestimento, sendo que o menor ângulo de contato é de $18^{\circ}$, obtido na amostra CR3, que contém $40 \%$ de resíduo no vidrado e $60 \%$ de resíduo na base cerâmica.

Palavras-chave: Resíduo; Rocha Ornamental; Vidrado; Molhabilidade.

\section{WETTABILITY TEST AS INDICATIVE OF THE POTENTIALITY TO USE DIEMENSION STONE WASTE IN COATING CERAMICS}

\begin{abstract} the ceramic base.

Keywords: Waste; Dimension Stone; Glaze; Wettability.

\section{INTRODUÇÃO}

Com destaque mundial no setor industrial de rochas ornamentais, o Brasil apresenta números significativos de produção e exportação destes materiais. Este setor tem crescido constantemente ao longo dos anos e apresenta grande perspectiva de crescimento futuro. Paralelamente,
\end{abstract}

The production of dimension stone plates generates a significant amount of wastes. The composition of these wastes shows the possibility of using them in several products, such as in manufacture of ceramic coating. The aim of this work was to study the wettability of glazed material on red ceramic, both composed with dimension stone waste. Wettability test was carried out using the Sessil Drop method, that evolve the fusion of glazed proof bodies on sintered red ceramic. The contact angle was measured during fusion of the glazed material and the results were treated through factorial $2^{2}$ analysis. The results showed that the wastes can be used for fabrication of ceramic coating, and the lowest contact angle is $18^{\circ}$, obtained in the CR3 sample, which contains $40 \%$ of waste in the glaze cover and $60 \%$ of waste in

aumenta também o volume de resíduos sólidos finos provenientes dos processos de extração e beneficiamento, durante os quais os blocos de rocha são transformados em chapas acabadas. Na maioria das vezes, esses resíduos não possuem destinação adequada, sendo armazenados em

'Programa de Pós-graduação em Engenharia Química, Universidade Federal do Espírito Santo - UFES, Campus de Alegre, Alto Universitário, Alegre, ES, Brasil.E-mail: tiagomarvilagomes@gmail.com

${ }^{2}$ Instituto de Tecnologia, Universidade Federal Rural do Rio de Janeiro - UFRRJ, Seropédica, RJ, Brasil. 
aterros ou depositados em lagos e rios, causando danos ambientais $[1,2]$.

A composição dos resíduos é bastante semelhante à de cerâmicas tradicionais e tem potencialidades para utilização como matéria-prima na fabricação de cerâmica de revestimento, sendo uma alternativa para diminuição da quantidade de material a ser descartado no ambiente [2-4]. Para se obter uma boa cobertura da placa cerâmica com o material vidrado, é necessário realizar estudos sobre as interações termodinâmicas, físicas e químicas entre o vidrado e a cerâmica de base [5].

O estudo do comportamento de molhabilidade é um importante parâmetro no processamento de materiais que apresentam a formação de fases líquidas em alta temperatura em junção com um sólido. A molhabilidade depende diretamente do balanço das energias interfaciais sólido-líquido-vapor do sistema [6,7]. No ensaio de molhabilidade, mede-se a variação do ângulo de contato $(\theta)$ entre a linha tangente à interface que separa o líquido e o vapor, e a linha paralela à superfície do sólido em função da temperatura e/ou do tempo. Considera-se que ocorre molhabilidade quando o ângulo de contato é menor que $90^{\circ}$ [8-10].

O método da Gota Séssil é o mais utilizado para a determinação da molhabilidade. Ele consiste em colocar o vidrado sobre o substrato de cerâmica vermelha e aquecer até a fusão do vidrado, medindo-se o ângulo de contato formado em função do tempo ou da temperatura $[6,1 \mathrm{I}, 12]$.

A molhabilidade pode ser classificada como molhabilidade física ou molhabilidade química, dependendo da natureza da força de ligação entre as interfaces. A molhabilidade física é possível por meio da dissociação completa ou parcial das ligações interatômicas dos sólidos. A força de Van der Waals é a energia atrativa para o molhamento físico. Na molhabilidade química ocorrem reações, nas quais a energia livre de Gibbs se torna a força motora para a diminuição da tensão interfacial sólido-líquido $[9,10]$.

A Equação I, denominada equação de Young, estabelece uma relação entre o ângulo de contato, a energia de superfície líquido-vapor $\left(\gamma^{\text {lv }}\right)$; a variação de energia livre de Gibbs no sistema $(\Delta \mathrm{G})$ representa a molhabilidade física [6].

$$
-\Delta G=\gamma^{l v}(1+\cos \theta)
$$

Para o molhamento químico, as reações alteram as condições de molhabilidade e a equação de Young precisa ser corrigida conforme a Equação 2, onde $\gamma^{\text {sv }}$ é a energia de superfície sólido-vapor, $\gamma^{\text {sL }}$ é a a energia de superfície

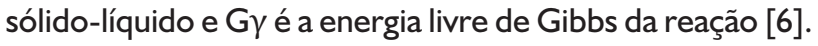

$$
\gamma^{S V}-\left(\gamma^{S L}+\frac{-d G \gamma}{d A d t}\right)=\gamma^{L V} \cos \theta
$$

Diversos estudos sobre molhabilidade foram feitos para diferentes sistemas e condições [13-16], porém, sobre vidrados em cerâmica vermelha é dificilmente encontrado na literatura, destacando a importância para cerâmica de revestimento.
O objetivo deste trabalho foi estudar a molhabilidade de vidrado sobre cerâmica vermelha, ambos compostos com diferentes porcentagens de resíduos finos produzidos pela serragem de rochas ornamentais processadas em teares com fios diamantados. Utilizou-se o planejamento fatorial $2^{2} \mathrm{com}$ repetição no ponto central, para identificar a composição do vidrado e da base cerâmica que possuem maior interação termodinâmica e que representam o melhor sistema para produzir cerâmica de revestimento.

\section{MATERIAIS E MÉTODOS}

Os resíduos de rochas ornamentais utilizados nos experimentos são oriundos de três tipos de granitos, cujos nomes comerciais são: Iberê Golden, Iberê Crema Bordeaux e Twilight, produzidos pela serragem de blocos utilizando tear com fios diamantados de uma empresa do município de Cachoeiro de Itapemirim - ES. A argila utilizada na base cerâmica foi obtida em uma indústria cerâmica localizada no município de Campos dos Goytacazes - RJ.

Para a produção das cerâmicas de base foram utilizadas composições denominadas AR40 (60\% de argila $+40 \%$ de resíduo), AR50 ( $50 \%$ de argila $+50 \%$ de resíduo) e AR60 (40\% de argila $+60 \%$ de resíduo). As composições AR40 e AR60 foram caraterizadas quanto às fases cristalinas utilizando Difratometria de Raios $X$.

Foram conformadas placas cerâmicas em prensa uniaxial nas dimensões de $120 \times 30 \times 5 \mathrm{~mm}$, com carga de prensagem de 2,5 toneladas. As placas foram sinterizadas a $1100^{\circ} \mathrm{C}$ por I hora em um forno elétrico com rampa de aquecimento de $20^{\circ} \mathrm{C} / \mathrm{min}$. Posteriormente elas foram submetidas ao ensaio de absorção de água segundo a norma NBR 13818 [17] e à determinação da densidade relativa. Tais parâmetros são proporcionais à porosidade, que é considerada propriedade importante no estudo da molhabilidade.

Nas composições dos vidrados, denominadas VI, V2 e V3, utilizou-se 40, 45 e $50 \%$ de resíduo de rochas ornamentais aditivados com fundentes e estabilizantes (Tabela I). O resíduo de rocha ornamental utilizado no experimento possui ponto de fusão de aproximadamente $1247^{\circ} \mathrm{C}$ [18]. Como normalmente o processo industrial de fabricação de cerâmica de revestimento emprega temperaturas próximas de $1100^{\circ} \mathrm{C}$, foi necessário adicionar fundentes ao resíduo.

A composição $\mathrm{V} 2$ utilizada neste trabalho foi proposta de acordo com diagrama ternário $\mathrm{Na}_{2} \mathrm{O}: \mathrm{CaO}: \mathrm{SiO}_{2}$ e composições da literatura [19]. Como fonte de sódio, cálcio e sílica foram utilizados $\mathrm{NaOH}, \mathrm{CaO}$ e o próprio resíduo contendo 7I,6\% de $\mathrm{SiO}_{2}$. As composições $\mathrm{VI}$ e V3 foram determinadas por meio de variação de $5 \%$ da concentração de resíduo proporcionalmente à variação dos outros constituintes, exceto o caulim e o carboximetilcelulose, os quais foram mantidos constantes. O caulim tem como finalidade principal promover a estabilidade das suspensões e 
o carboximetilcelulose melhora a viscosidade na preparação do vidrado.

O pó do vidrado foi compactado em prensa hidráulica utilizando-se uma matriz de aço com dupla ação de pistões. A força uniaxial utilizada foi de 2,5 toneladas. $O$ corpo de prova foi cortado com dimensões aproximadas de $3 \times 3 \times 3 \mathrm{~mm}$.

Foi utilizado o método da Gota Séssil para o estudo da molhabilidade. $O$ vidrado foi colocado sobre uma placa de $30 \times 30 \times 5 \mathrm{~mm}$ de cerâmica vermelha previamente sinterizada, e ambos foram aquecidos até a fusão do vidrado.

O ensaio de molhabilidade foi registrado por uma câmera fotográfica digital e o ângulo de contato visualizado em cada imagem foi medido, utilizando o software ImageJ e a ferramenta Angle Tool. Curvas do perfil de molhabilidade em relação à temperatura e tempo foram plotadas em gráficos.

As amostras conformadas para o ensaio de molhabilidade foram denominadas CRI, CR2, ... e CR9, compostas pelas combinações das bases cerâmicas (AR40, AR50 e AR60) com os vidrados (VI, V2 e V3) (Figura I). Assim, CRI representa uma cerâmica de base contendo $40 \%$ de resíduo (AR40) e vidrado de composição VI; CR2, CR3 e CR4 também representam os ensaios de molhabilidade em função da temperatura, com diferentes bases cerâmicas e vidrados. As amostras CR5 a CR9 são os pontos centrais, utilizados para análise do erro.

Para o entendimento do planejamento experimental é necessário definir alguns conceitos iniciais [20,2l], conforme descrito a seguir:

Tabela I. Composição dos vidrados

\begin{tabular}{crrr}
\hline Composição (\% m/m) & V I & V2 & V3 \\
\hline Resíduo & 40,0 & 45,0 & 50,0 \\
$\mathrm{NaOH}$ & 28,5 & 25,9 & 23,2 \\
$\mathrm{CaO}$ & $1 \mathrm{I}, 5$ & 10,2 & $9, \mathrm{I}$ \\
$\mathrm{KOH}$ & 0,9 & 0,8 & 0,7 \\
$\mathrm{Al}_{2} \mathrm{O}_{3}$ & 0,8 & 0,7 & 0,6 \\
$\mathrm{H}_{3} \mathrm{BO}_{3}$ & 10,8 & 9,9 & 8,9 \\
$\mathrm{Caulim}_{\text {Carboximetilcelulose }}$ & 7,0 & 7,0 & 7,0 \\
& 0,5 & 0,5 & 0,5 \\
\hline
\end{tabular}

\begin{tabular}{ccc}
\hline Amostras & Base cerâmica & Vidrado \\
\hline CR1 & AR40 & V1 \\
CR2 & AR40 & V3 \\
CR3 & AR60 & V1 \\
CR4 & AR60 & V3 \\
CR5 & AR50 & V2 \\
CR6 & AR50 & V2 \\
CR7 & AR50 & V2 \\
CR8 & AR50 & V2 \\
CR9 & AR50 & V2
\end{tabular}

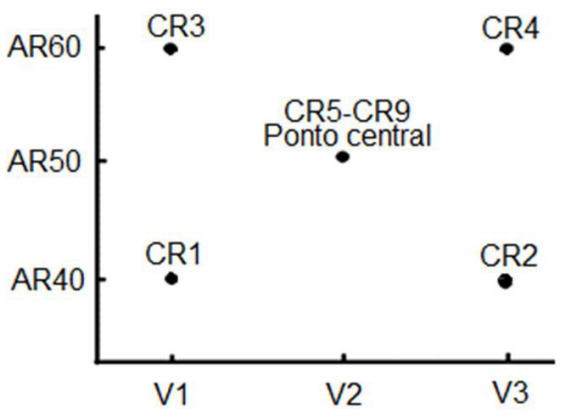

Figura I. Composição das amostras CRI, CR2, ..., CR9 de acordo com a base cerâmica e o vidrado utilizados e planejamento dos experimentos de molhabilidade em função da temperatura. 


\section{RESULTADOS E DISCUSSÃO}

A Figura 2 mostra o difratograma de raios $X$ das composições AR40 e AR60, que são os níveis (-) e (+) do fator $c_{1}$, respectivamente. A composição AR50 é similar e com intensidade intermediária de picos entre AR40 e AR60. Foram identificados picos característicos de quartzo $\left(\mathrm{SiO}_{2}\right)$, biotita $\left(\mathrm{K}(\mathrm{Mg}, \mathrm{Fe})_{3}(\mathrm{OH}, \mathrm{F})_{2}(\mathrm{Al}, \mathrm{Fe}) \mathrm{Si}_{3} \mathrm{O}_{10}\right)$, caulinita $\left(\mathrm{Al}_{2} \mathrm{Si}_{2} \mathrm{O}_{5}(\mathrm{OH})_{4}\right)$ e dos fesidspatos, albita $\left(\mathrm{NaAlSi}_{3} \mathrm{O}_{8}\right)$ e microclina $\left(\mathrm{KAISi}_{3} \mathrm{O}_{8}\right)$.

A Tabela 2 mostra os valores de massa específica real $(\rho)$, massa específica aparente $\left(\rho_{\mathrm{a}}\right)$, densidade relativa (DR) e absorção de água (Abs) para as composições AR40, AR50 e AR60. Os valores de densidade relativa de aproximadamente $78 \%$ indicam que as cerâmicas de base são compactas e possuem aproximadamente $22 \%$ de porosidade. A absorção de água, que é uma propriedade inversamente proporcional à porosidade, foi de aproximadamente $10 \%$, indicando a possibilidade de existência de porosidade fechada nas cerâmicas. Segundo ABNT NBR I 38I8 (1997), as cerâmicas com aproximadamente $10 \%$ de absorção de água são indicadas para revestimento de parede [17]. Desta forma, pode-se considerar que a porosidade nas cerâmicas de base é aceitável para os produtos normalmente destinados, e possivelmente não interfe nos ensaios de molhabilidade, garantindo a reprodutividade dos resultados.

O efeito da temperatura na molhabilidade do vidrado sobre a cerâmica vermelha pode ser observado nas Figuras 3 e 4 . As imagens da Figura 3 são da amostra
CRI, mas representam os demais ensaios de molhabilidade por apresentarem comportamento semelhante. A Figura 4 apresenta a variação do ângulo de contato com a temperatura para as amostras CRI, CR2, CR3 e CR4 e para os pontos centrais (CR5 a CR9, para obtenção da média e desvio padrão).

Todas as curvas da Figura 4 apresentam a diminuição do ângulo de contato com a temperatura, assim como descrito em outros trabalhos que utilizam sistemas diversos $[8,22,23]$. Nas amostras CRI e CR3, que possuem a cerâmica de base com respectivamente 40 e $60 \%$ de resíduo e o vidrado $\mathrm{VI}$ (com maior porcentagem de fundentes no resíduo), o ângulo de contato final foi menor (na temperatura de $1100^{\circ} \mathrm{C}$ ) do que nas demais amostras (CR2, CR4 e Ponto Central). Isto indica que a quantidade de fundente no vidrado tem influência significativa na molhabilidade em cerâmica vermelha. As curvas CRI e CR3 apresentam comportamento de molhabilidade semelhante, sendo que a CR3 tem espalhamento ligeiramente superior ao da amostra CRI. Isto é atribuído à maior porcentagem de resíduo na composição da cerâmica de base, que proporciona maior afinidade química ao vidrado, por semelhança de composição. Esta similaridade também é observada nas amostras CR2 e CR4, e novamente, a CR4 apresenta espalhamento ligeiramente superior ao da amostra CR2, por possuir maior quantidade de resíduo na composição da cerâmica vermelha.

Vale ressaltar que os resíduos de rochas ornamentais possuem silicatos hidratados e feldspatos em sua composição. Esses feldspatos atuam como fundentes na cerâmica de base

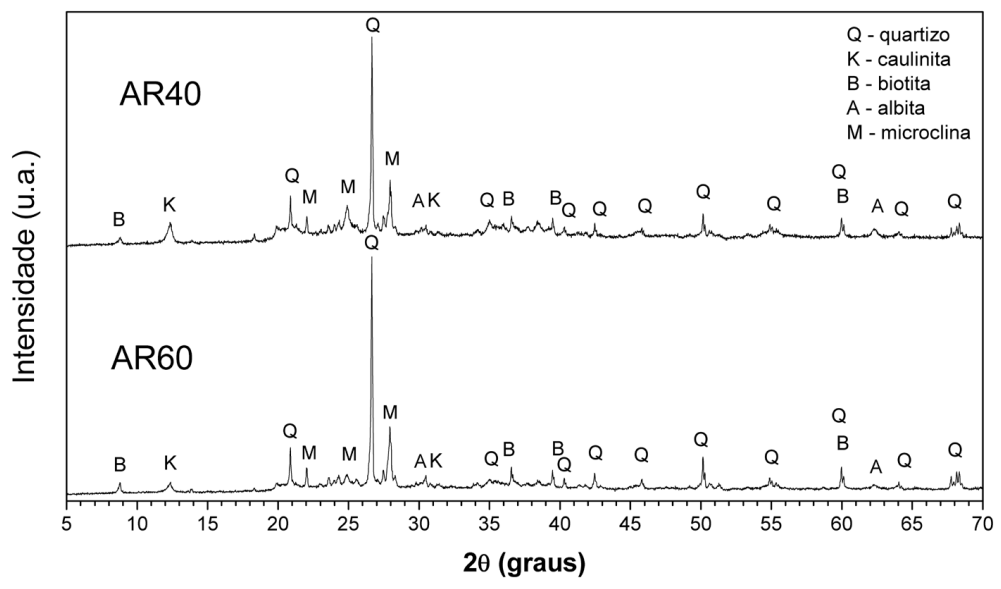

Figura 2. Difratograma de Raios $X$ das composições AR40 e AR60.

Tabela 2. Massa específica real $(\rho)$, massa específica aparente $\left(\rho_{\mathrm{a}}\right)$, densidade relativa (DR) e absorção de água (Abs) das composições AR40, AR50 e AR60

\begin{tabular}{cccc}
\hline Composições & AR40 & AR50 & AR60 \\
\hline$\rho\left(\mathrm{g} / \mathrm{cm}^{3}\right)$ & $2,35 \mathrm{I}$ & 2,355 & 2,355 \\
$\rho_{\mathrm{a}}\left(\mathrm{g} / \mathrm{cm}^{3}\right)$ & 1,827 & 1,833 & 1,835 \\
$\mathrm{DR}(\%)$ & 77,8 & 78,0 & 78,1 \\
Abs $(\%)$ & $\mathrm{II}, 5 \mathrm{I}$ & 10,02 & 9,12 \\
\hline
\end{tabular}



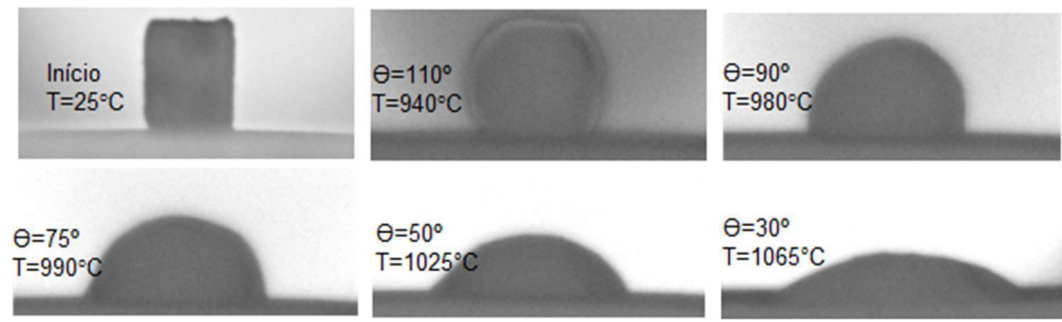

$\theta=30^{\circ}$ $\mathrm{T}=1065^{\circ} \mathrm{C}$

Figura 3. Imagens da evolução do formato da gota de vidrado sobre uma placa de cerâmica vermelha durante os ensaios de molhabilidade para a amostra CRI.

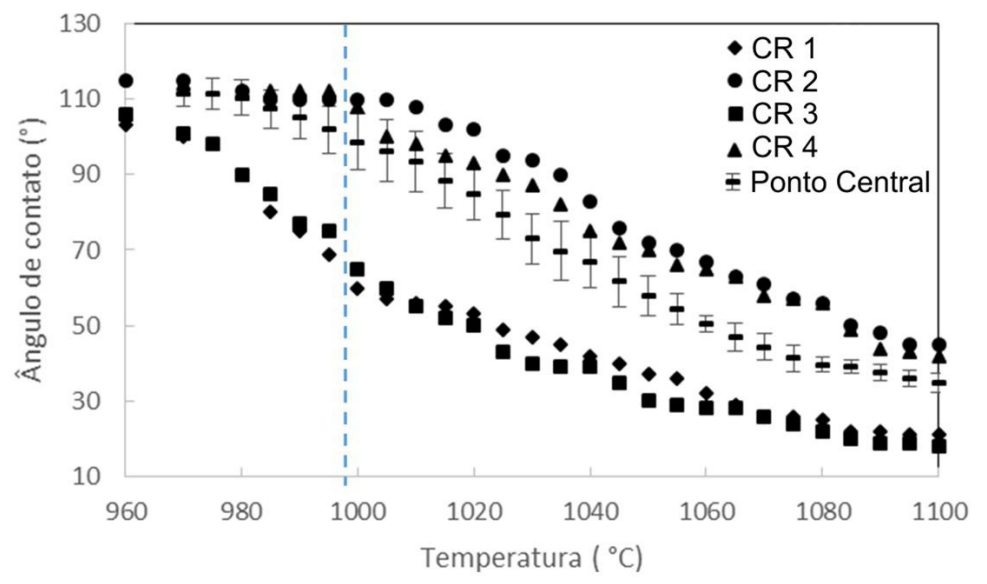

Figura 4. Variação do ângulo de contato em função da temperatura para as amostras CRI, CR2, CR3, CR4 e Ponto Central (a linha azul tracejada indica a isotérma utilizada no experimento de molhabilidade em função do tempo).

e, devido à sua boa mobilidade atômica, melhora a interação com o vidrado fundido durante o ensaio de molhabilidade, mesmo que não tão representativo nos resultados finais quanto a influência da concentração de resíduos no vidrado.

Os feldspatos agregam ao vidrado os denominados modificadores. Os modificadores são componentes de ligação iônica ( $\mathrm{CaO}$ e $\mathrm{NaOH})$, que proporcionam diminuição na temperatura de fusão por promover a descontinuidade na rede dos vidroformadores e a redução da viscosidade, melhorando o espalhamento do vidrado. Os vidroformadores $\left(\mathrm{SiO}_{2}, \mathrm{Al}_{2} \mathrm{O}_{3}\right.$ e $\mathrm{H}_{3} \mathrm{BO}_{3}$ ) possuem elevadas forças de ligação covalente. Desta forma, ao diminuir a concentração de resíduo, que é majoritariamente composto por $\mathrm{SiO}_{2}$, no vidrado, pode-se observar a diminuição do ângulo de contato para uma certa temperatura.

A Tabela 3 mostra os resultados referentes à ANOVA para os ângulos de contato finais do experimento de molhabilidade em função da tempertaura para as 9 amostras estudadas, com um nível de significância de 0,95 . Foram analisados os fatores concentração de resíduo na base cerâmica, concentração de resíduo no vidrado e a interação entre ambos os fatores. $O$ fator $c_{1}$, concentração de resíduo na base cerâmica, apresentou baixo valor de $F\left(F_{\text {calc }}=7,77\right)$ e pouca significância em relação ao ângulo de contato, enquanto o fator $c_{2}$, concentração de resíduo no vidrado, apresentou alto valor de $F\left(F_{\text {calculado }}=350,63\right)$ e, consequentemente, influência estatisticamente significativa na molhabilidade. Foram também obtidos valores de $F_{\text {calculado }}$ para curvatura e interação entre as variáveis $\left(c_{2} c_{1}\right)$ menores que os tabelados, indicando, respectivamente, que se trata de um fenômeno de primeira ordem e que não há interação entre as variáveis.

Para se obter um modelo empírico para predição do fenômeno estudado, foi proposto um modelo linear, representado pela Equação 3, onde a corresponde ao ângulo de contato e $\varepsilon$ corresponde ao erro aleatório experimental. Os parâmetros $\alpha_{0}, \alpha_{1}$ e $\alpha_{2}$ são do modelo, e as variáveis $c_{1}$ e $c_{2}$ representam os fatores.

$$
\boldsymbol{a}=\alpha_{0}+\alpha_{1} c_{1}+\alpha_{2} c_{2}+\varepsilon
$$

Para determinar os valores dos parâmetros deve-se ajustar a Equação 3 aos valores (ângulo de contato, $c_{1}, c_{2}$ ) obtidos em cada experimento (por exemplo, CRI possui ângulo final $\theta_{1100^{\circ} \mathrm{C}}=21^{\circ}, c_{1}=60 \%$ e $\left.c_{2}=40 \%\right)$. Isto é, necessita-se resolver um sistema de 9 equações, onde cada equação contém valores de ângulo, $c_{1}, c_{2}$, e cujas incógnitas são $\alpha_{0}, \alpha_{1}$ e $\alpha_{2}$. Esse sistema pode ser representado de forma compacta por uma única equação matricial genérica (Equação 4), 
Tabela 3. ANOVA das variáveis independentes

\begin{tabular}{|c|c|c|c|c|c|}
\hline & $\begin{array}{l}\text { Soma dos } \\
\text { Quadrados }\end{array}$ & GL & Média da Soma & $\mathbf{F}_{\text {calculado }}$ & $\mathbf{F}_{\text {tabelado }}$ \\
\hline$c_{1}$ & 12,25 & 1 & 12,25 & 7,777778 & 12,22 \\
\hline$c_{2}$ & 552,25 & 1 & 552,25 & 350,6349 & \\
\hline$c_{2} c_{1}$ & 0,25 & 1 & 0,25 & 0,15873 & \\
\hline Curvatura & 18,05 & 1 & 18,05 & 11,46032 & \\
\hline Erro & 6,3 & 4 & $\mathrm{I}, 575$ & & \\
\hline Total & 589,1 & 8 & 73,6375 & & \\
\hline
\end{tabular}
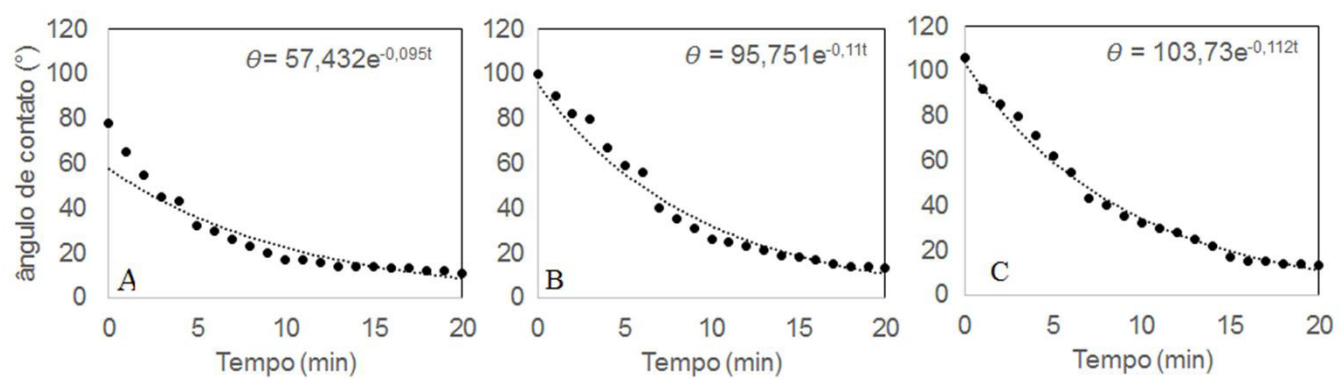

Figura 5. Ângulo de contato em função do tempo para o vidrado A) VI, B) V2 e C) V3 em cerâmica de base com $50 \%$ de resíduo, em temperatura de $1000^{\circ} \mathrm{C}$ por $20 \mathrm{~min}$.

$$
A=X B+e
$$

$$
\text { onde, } \mathrm{A}=\left[\begin{array}{l}
\alpha 1 \\
\alpha 2 \\
\ldots \\
\alpha_{9}
\end{array}\right], \mathrm{X}=\left[\begin{array}{ccc}
1 & X_{1} & X_{1}^{2} \\
1 & X_{2} & X_{2}^{2} \\
\ldots & \ldots & \ldots \\
1 & X_{9} & X_{9}^{2}
\end{array}\right], \mathrm{B}=\left[\begin{array}{c}
\alpha_{0} \\
\alpha_{1} \\
\alpha_{2}
\end{array}\right], \mathrm{e}=\left[\begin{array}{c}
\varepsilon_{1} \\
\varepsilon_{2} \\
\ldots \\
\varepsilon_{9}
\end{array}\right]
$$

A maneira tradicional de conseguirmos ajustar uma reta aos pontos experimentais é localizá-la de tal maneira que a soma dos quadrados dos resíduos seja mínima, razão pela qual esse método é chamado de ajuste por mínimos quadrados. Para que o valor do somatório do erro seja mínimo, é preciso que suas derivadas em relação a $\alpha_{0}, \alpha_{1}$ e $\alpha_{2}$ se anulem. A partir das equações obtidas, pode-se calcular $\alpha_{0}, \alpha_{1}$ e $\alpha_{2}$ resolvendo uma única equação matricial (Equação 5), onde a matriz $\mathbf{X}^{t}$ é a matriz $\mathbf{X}$ transposta.

$$
B=\left(X^{t} X\right)^{-1} X^{t} A
$$

Obtendo-se os valores de $\alpha_{0}, \alpha_{1}$ e $\alpha_{2}$ ao resolver a Equação 5 pode-se, enfim, reescrever a Equação 4, como a Equação 6, que representa a molhabilidade em função das concentrações de resíduo no vidrado e na base cerâmica na temperetura de $1100^{\circ} \mathrm{C}$.

$$
B=\left[\begin{array}{c}
33 \\
1,75 \\
11,75
\end{array}\right] \text {, então } a=33-I, 75 x_{1}+1 /, 75 x_{2}
$$

Pode-se observar que o parâmetro $\alpha_{2}$ se apresenta 6,7 vezes maior que o parâmetro $\alpha_{1}$, mostrando ter maior interferência na variável resposta, convalidando os resultados da Tabela 3.

A análise dos resíduos é fundamental para avaliar a qualidade do ajuste de qualquer modelo matemático. $O$ método mais usado para essa avaliação numérica é a Análise da Variância do Modelo, mostrada na Tabela 4. O R ${ }^{2}$ é obtido pela Equação 7 , o qual indica que $88,57 \%$ da variação é explicada pelo modelo matemático, se mostrando um modelo preditivo eficaz.

$$
R^{2}=\frac{\text { Soma dos quadrados da regressão }}{\text { Soma dos quadrados total }}
$$

A Figura 5 apresenta o comportamento do ângulo de contato em função do tempo para $\mathrm{VI}, \mathrm{V} 2 \mathrm{e} \mathrm{V3}$ em uma cerâmica de base com $50 \%$ de resíduo, na isoterma de $1000^{\circ} \mathrm{C}$. Neste caso, o ensaio de molhabilidade do vidrado VI sobre AR50 equivale a um valor aproximadamente intermediário entre CRI e CR3. Analogamente, V2 equivale aos ensaios de CR5 a CR9, e V3 ao valor intermediário de CR2 e CR4. Observa-se que a redução do ângulo de contato é maior nos primeiros minutos, com um comportamento exponencial, até que o equilíbrio seja atingido em aproximadamente 20 minutos. Esse mesmo comportamento é observado em trabalhos da literatura que utilizam outros sistemas $[8,22,23]$. Os valores de ângulo de contato final da Figura 5 foram próximos aos obtidos nos experimentos de molhabilidade em função da 
Tabela 4. ANOVA da regressão linear

\begin{tabular}{cccc}
\hline & Soma dos Quadrados & GL & Média da Soma \\
\hline Regressão & 574,4856 & 2 & 287,2428 \\
Resíduos & 37,0485 & 6 & 6,17475 \\
Falta de ajuste & 11,8485 & 2 & 5,92425 \\
Erro & 25,2 & 4 & 6,3 \\
Total & 648,5826 & 8 & 81,07283 \\
\hline
\end{tabular}

temperatura (Figura 4) para as amostras CRI e CR3, mas as demais amostras apresentaram maiores valores. Porém, cabe ressaltar que a $1000^{\circ} \mathrm{C}$ (linha vertical tracejada na Figura 4), os ângulos de contato são equivalentes ao do início das curvas da Figura 5 , ou seja, para $\mathrm{t}=0$, sendo que $\mathrm{VI}$ apresenta valor ligeiramente menor. Isto mostra que, embora termodinamicamente o menor valor de ângulo de contato ocorra a $1100^{\circ} \mathrm{C}$ no gráfico de molhabilidade em função da temperatura (Figura 4), estas amostras poderiam obter estes mesmos ângulos de contato, ou até inferiores, quando submetidos a $1000^{\circ} \mathrm{C}$, porém, após 20 minutos, conforme mostrado nas respectivas equações indicadas na Figura 5.

Pelas curvas de molhabilidade mostradas na Figura 4, pode-se identificar qual temperatura é mais interessante para ocorrer o espalhamento do vidrado sobre a cerâmica vermelha. Na Figura 5 pode-se inferir quanto tempo levaria para se obter o mesmo espalhamento numa determinada temperatura, trazendo oportunidades de escolhas mais adequadas (Composição/Temperatura/ Tempo) para o processamento industrial de cerâmicas de revestimento.

\section{CONCLUSÕES}

As amostras estudadas apresentaram boa molhabilidade do vidrado na cerâmica vermelha, ambos compostos de resíduos finos de rochas ornamentais serradas em tear de fio diamantado, indicando a potencialidade de utilização deste tipo de resíduo para aplicação em cerâmica de revestimento. $\mathrm{O}$ aumento da concentração de fundente no vidrado promove uma maior molhabilidade. A variação na concentração de resíduo na base cerâmica apresenta baixa influência na molhabilidade, mas este foi considerado um fator positivo, pois apresentou ligeira melhora no espalhamento, devido à interação química dos componentes. $\mathrm{A}$ amostra CR3, que contém $40 \%$ de resíduo no vidrado e $60 \%$ de resíduo na base cerâmica, apresentou o menor ângulo de contato $\left(18^{\circ}\right)$, sendo esta composição a mais indicada para cerâmica de revestimento.

\section{Agradecimentos}

Os autores agradecem ao CNPq N $550307 / 201$ I-7, e à FAPES $N^{\circ} 53635230 / 201$ I, pelo apoio financeiro.

\section{REFERÊNCIAS}

I Associação Brasileira da Indústria de Rochas Ornamentais - ABIROCHAS. São Paulo: Informe 07 (2016).

2 Reis AS, Della-Sagrillo VP, Velenzuela-Diaz FR. Analisys of dimension stone waste addition clayey mass used in roof tile production. Materials Research. 2015; 18:63-69.

3 Rodrigues GF, Alves JO, Tenório JAS, Espinosa DCR. Estudo de resíduos de rochas ornamentais para produção de materiais vítreos. Tecnologica em Metalurgia, Materiais e Mineração. 201 I;8:203-21 I.

4 Taguchi SP, Santos JC, Gomes TM, Cunha NA. Avaliação das propriedades tecnológicas de cerâmica vermelha incorporada com resíduo de rocha ornamental proveniente do tear de fio diamantado. Cerâmica. 2014;60:291-296.

5 Wolf FG. Modelagem da interação fluido-sólido para simulação de molhabilidade e capilaridade usando o modelo lattice-boltzmann [Tese]. Florianópolis: Universidade Federal de Santa Catarina; 2006.

6 Luz AP, Ribeiro S, Pandolfelli VC. Uso da molhabilidade na investigação do comportamento de corrosão de materiais refratários. Cerâmica. 2008;54:174-I83.

7 Alghunaim A, Kirdponpattara S, Newby BZ. Techniques for determining contact angle and wettability of powders. Powder Technology. 2016;287:20I-2I5.

8 Motta FV, Balestra RM, Ribeiro S, Taguchi SP. Wetting behaviour of SiC ceramics: Part I $-\mathrm{E}_{2} \mathrm{O}_{3} / \mathrm{Al}_{2} \mathrm{O}_{3}$ additive system. Materials Letters. 2004;58:2805-2809.

9 Wang Z, Elimelech M, Lin S. Environmental appliactions of interfacial materials with special wettability. Environmental Science \& Technology. 2016;50:2132-2150. 
I0 Bigheti CMM, Ribeiro S, Taguchi SP, Strecker K, Machado JPB, Santos C. Characterization of rare earth oxide-rich glass applied to the glass-infiltration of a ceramic system. Ceramics International. 2014;40:1619-1625.

II Ribeiro S, Taguchi SP, Motta FV, Balestra RM. The wettability of $\mathrm{SiC}$ ceramics by molten $\mathrm{E}_{2} \mathrm{O}_{3}(\mathrm{ss}) / \mathrm{AIN}$ $\left(\mathrm{E}_{2} \mathrm{O}_{3}(\mathrm{ss})=\right.$ solid solution of rare earth oxides. Ceramics International. 2007;33:527-530.

12 Luz AP, Ribeiro S, Domiciano VG, Pandolfelli VC. Slag melting temperature and contact angle on high carbon containing refractory substrates. Cerâmica. 20I I;57(342): I 40- I 49.

13 Xu Q, Guo L, Zhang L, Liu H. Wettability of zirconium-coated alumina by molten aluminum. Surface and Coatings Technology. 2016;302:150-157.

14 Shen P, Zhang L, Wang Y. Wettability between molten slag and dolomitic refractory. Ceramics International. 2016;42:16040-16048.

15 Fu W, Song XB, Zhao YX, Cao J, Feng JC, Jin C, et al. Effect of Ti content on the wetting behavior of Sn0.3Ag0.7Cu/ AIN system. Materials \& Design. 2017;1 15:I-7.

16 Shen p, Fujii H, Nogi K. Wetting mechanism of AgCuTi on heterogeneous surface of Diamond/Cu composites. Surface and Coatings Technology. 2017;325:I559-1569.

17 Associação Brasileira de Normas Técnicas. NBR I38|8: Placas cerâmicas para revestimento - especificação e métodos de ensaios. Rio de Janeiro: ABNT; 1997.

18 Taguchi SP, Cunha JA, Cunha NA, Santos FA, Silva AC. Produção de vidrado a partir de resíduo de rochas ornamentais. In: Anais do $20^{\circ} \mathrm{CBECIMAT}$ - Congresso Brasileiro de Engenharia e Ciência dos Materiais; 2012 November 04-08; Joinville, Brasil. São Paulo: Metallum; 2012. p. 106-I38.

19 Sorli S, Tena MA, Mestre A, Lusar M, Monós G. Efeito da fase principal devitrificada sobre a microestrutura e as propriedades mecânicas de vidrados cerâmicos. Cerâmica Industrial. 2005; I0:6-13.

20 Barros Neto B, Scarminio IS, Bruns RE. Como fazer experimentos. 2.ed. Campinas: Editora Unicamp; 200 I.

2I Cunico MWM, Cunico MM, Miguel OG, Zawadzki SS. Planejamento fatorial: Uma ferramenta valiosa para definição de parâmetros experimentais na pesquisa. Visão Acadêmica. 2008;9:23-32.

22 Luz AP, Ribeiro S. Wetting behaviour of silicone nitride ceramics by Ti-Cu alloys. Ceramics International. 2006;34:305-309.

23 Eustathopoulos N, Sobczak N, Passerone A, Nogi K. Measurement of contact angle and work of adhesion at high temperature. Journal of Materials Science. 2005;40:227I-2280.

Recebido em: I Jun. 2017

Aceito em: II Jun. 2018 